\section{Despesas municipais em atenção primária à saúde no Rio Grande do Sul, Brasil: um estudo ecológico}

\author{
Municipal spending on primary health care in Rio \\ Grande do Sul State, Brazil: an ecological study
}

\author{
Gastos municipales en atención primaria de \\ salud en Río Grande do Sul, Brasil: \\ un estudio ecológico
}

\section{Resumo}

O objetivo do estudo é explorar a associação entre componentes do Piso de Atenção Básica (PAB) fixo e variável, fatores sociodemográficos e perfil epidemiológico com as despesas municipais em atenção primária à saúde no Rio Grande do Sul, Brasil. Foi realizado estudo ecológico com 496 municípios do Rio Grande do Sul. A variável despesa média municipal dos anos de 2011 a 2013 do bloco financeiro da atenção primária à saúde que representou as despesas efetivas com o repasse de recursos federais foi extraída do Relatório Gerencial da Sala de Apoio à Gestão Estratégica. Utilizou-se modelo de regressão linear múltipla. Para fins de ajuste do modelo, as variáveis foram agrupadas em cinco blocos de acordo com o objetivo do estudo. A despesa média com atenção primária à saúde foi de $R \$ 81,20(D P \pm 35,50)$ por habitante-ano. $O$ bloco de variáveis que compõem o PAB fixo explicou $39 \%\left(R^{2}=0,39\right) d a$ variabilidade de despesas entre municípios, enquanto que, no bloco do $P A B$ variável, o $R^{2}$ foi 0,82, no bloco sociodemográficas, o $R^{2}$ foi 0,26, no bloco de estrutura-desempenho, o $R^{2}$ foi 0,46, e, no bloco de perfil epidemiológico, o $R^{2}$ foi 0,15 . No modelo final, a variável que esteve associada a maiores valores estimados de gasto com atenção primária à saúde foi a taxa de equipes de saúde da família. Municípios com o número de equipes entre 135 e 41 por 100 mil habitantes-ano possuem um gasto de $R \$ 51,00$ per capita a mais do que municípios com o número de equipes entre 0 e 8. Despesa em atenção primária à saúde parece estar mais atrelada às politicas federais de indução do que a fatores associados com a demanda em saúde, como o perfil demográfico e epidemiológico dos municípios do Rio Grande do Sul.

Financiamento da Atenção à Saúde; Recursos Financeiros em Saúde; Planejamento em Saúde; Gestão em Saúde; Atenção Primária à Saúde
Fabiana da Silva Cabreira 1,2

Fernando Ritter 2,3

Violeta Rodrigues Aguiar 2,3

Roger Keller Celeste 2

doi: 10.1590/0102-311X00150117

\author{
Correspondência \\ F. S. Cabreira \\ Instituto Federal de Educação, Ciência e Tecnologia Farroupilha. \\ RS-377, Km 27, Alegrete, RS 97555-000, Brasil. \\ fabiana.cabreira@iffarroupilha.edu.br
1 Instituto Federal de Educação, Ciência e Tecnologia Farroupilha, Alegrete, Brasil.
2 Faculdade de Odontologia, Universidade Federal do Rio Grande do Sul, Porto Alegre, Brasil.
3 Secretaria Municipal de Saúde de Porto Alegre, Porto Alegre, \\ Brasil.
}




\section{Introdução}

O sistema público de saúde no Brasil, assim como em outros países, possui recursos financeiros limitados. Países cujos sistemas de saúde são mais orientados à atenção primária à saúde alcançam melhores níveis de saúde, maior satisfação com os serviços de saúde e menores custos dos serviços em geral ${ }^{1}$. Logo, entende-se a atenção primária como nível de cuidado estratégico capaz de atingir ampla cobertura e resolutividade.

No Brasil, o financiamento em atenção primária à saúde, bem como dos outros níveis de atenção, provém, principalmente, de transferências do governo federal para os municípios. A Norma Operacional Básica de 1996 estabeleceu o, atualmente chamado, Piso de Atenção Básica fixo (PAB fixo), em que os valores a serem transferidos eram basicamente definidos pela multiplicação de valor per capita nacional pela população de cada município 2. Em 2011, os municípios foram divididos em quatro faixas de financiamento, por meio de cálculo que inclui as variáveis: porte populacional, PIB per capita, percentual da população com plano de saúde, percentual da população com bolsa família ou percentual da população em extrema pobreza e densidade demográfica 3. O PAB fixo deve cobrir a demanda por serviços e a manutenção da estrutura instalada, por exemplo, recursos humanos, equipamentos e unidades de saúde. Baseando-se nessa metodologia, espera-se que tais variáveis que interferem na distribuição das receitas também interfiram nas despesas municipais associadas com a demanda pela atenção primária à saúde.

Além do PAB fixo, também passaram a compor o financiamento em atenção primária à saúde os incentivos vinculados à adesão e a manutenção de programas definidos em nível federal e, posteriormente, transformados em Piso de Atenção Básica variável (PAB variável) 2. A adesão a políticas/ programas do $\mathrm{PAB}$ variável pode ser outra fonte de receita, As principais iniciativas estão atreladas às seguintes iniciativas: Estratégia Saúde da Família (ESF), agentes comunitários de saúde (ACS), equipes de saúde bucal na ESF (ESB) e Núcleos de Saúde da Família (NASF).

É esperado que as receitas municipais sejam suficientes para cobrir os gastos dos serviços de saúde, entretanto, há uma série de fatores que afetam a demanda por esses serviços em nível municipal e que não estão contempladas nas receitas incluídas no cálculo do PAB fixo. As características demográficas, sociais, epidemiológicas: população de crianças, idosos e mulheres; escolaridade; renda domiciliar; número de óbitos por causas externas, número de óbitos por diabetes mellitus, número de óbitos infantis e nascidos vivos de baixo peso, podem ser consideradas importantes fatores. Diversos autores relatam que muitas dessas variáveis estão relacionadas com a utilização de serviços 4,5,6,7,8,9 e devem ser avaliadas por sua provável relação com as despesas em atenção primária à saúde.

Não se conhece estudos que expliquem por que um município gasta menos que outro em atenção primária à saúde. Além disso, questiona-se se os fatores de regulação federal elencados são suficientes para dar conta das especificidades municipais, se são relevantes em promover equidade no financiamento em atenção primária à saúde. Dessa forma, o objetivo deste estudo é explorar a associação entre as variáveis que definem o PAB fixo e variável, fatores sociodemográficos, fatores de estrutura e desempenho e perfil epidemiológico municipal com as despesas municipais em atenção primária à saúde do Rio Grande do Sul no período de 2011 a 2013.

\section{Métodos}

Este é um estudo ecológico, de caráter exploratório, com os 496 municípios do Estado do Rio Grande do Sul que possuíam informação de despesa em atenção primária à saúde para os anos estudados. Os dados foram oriundos de vários sistemas de informação: Cadastro Nacional de Estabelecimentos de Saúde (CNES); Sistema de Informações sobre Mortalidade (SIM); Sistema de Informações sobre Nascidos Vivos (SINASC); Agência Nacional de Saúde Suplementar (ANS); Ministério do Desenvolvimento Social e Combate à Fome. Também foram extraídos dados do Atlas de Desenvolvimento Humano no Brasil (ATLAS) 10, do Instituto Brasileiro de Geografia e Estatística (IBGE, http://www.ibge.gov.br) e da Sala de Apoio à Gestão Estratégica (SAGE, http://sage.saude.gov.br/).

A variável de desfecho foi a despesa média municipal dos anos de 2011 a 2013 do bloco financeiro da atenção básica. Esses dados foram extraídos do Relatório Gerencial da Sala de Apoio à Gestão 
Estratégica no período de 28 a 30 de outubro de 2014. Essa variável representa os gastos em atenção primária à saúde efetivados pelos municípios com o repasse de recursos federais.

As variáveis exploratórias foram agrupadas em cinco blocos: $\mathrm{PAB}$ fixo, $\mathrm{PAB}$ variável, sociodemográficas, estrutura e desempenho e perfil epidemiológico. No bloco PAB fixo, as variáveis incluídas foram: PIB (Produto Interno Bruto) per capita médio dos anos de 2010 a 2012, anos que antecedem o desfecho; densidade demográfica (extraída do censo do IBGE do ano de 2010); percentual médio de beneficiários do Programa Bolsa Família por município dos anos de 2013 e 2014 (anos disponíveis no Ministério do Desenvolvimento Social e Combate à Fome); percentual médio de beneficiários de planos de saúde privado em relação à população por município dos anos de 2010 a 2012, dados da ANS; percentual da população vivendo em extrema pobreza por município do ano de 2010, disponível no Ministério do Desenvolvimento Social e Combate à Fome; e porte populacional, médias dos anos de 2010 a 2012 do IBGE. No bloco PAB variável, foram incluídas as variáveis: taxa de ESF por 10 mil habitantes-ano; taxa de ACS por 10 mil habitantes-ano; proporção de ESB por ESF e proporção de NASF por ESF. Os dados brutos dessas variáveis foram obtidos do CNES e calculados a partir da média dos anos de 2010 a 2012. Do bloco das variáveis sociodemográficas, fazem parte as variáveis: renda domiciliar per capita; Índice de Desenvolvimento Humano Municipal (IDHM); escolaridade calculada a partir do percentual de pessoas com 18 anos ou mais com Ensino Médio completo do total de habitantes com mais de 18 anos de cada município; índice Gini; percentual de mulheres; percentual de idosos (com 60 anos ou mais) e percentual de crianças (de 0 a 5 anos). A renda domiciliar per capita, o IDHM e a escolaridade foram obtidos do ATLAS 10, sendo os dados do ano de 2010. O índice Gini e o número de habitantes por faixa etária e sexo foram obtidos do IBGE usando-se as médias dos anos de 2010 a 2012. Compõem o bloco estrutura e desempenho as variáveis: o Índice de Desempenho do SUS (IDSUS); taxa de unidades básicas de saúde (UBS) por 10 mil habitantes-ano e taxa de hospitais- dia, gerais e especializados por 10 mil habitantes-ano. Do CNES, foram obtidos, para os anos de 2010 a 2012, o número de UBS e o de hospitais. Da SAGE, foi obtido o IDSUS do ano de 2010, O último bloco é o perfil epidemiológico em que foram incluídas as variáveis: taxa de mortalidade por causas externas e taxa de mortalidade por diabetes mellitus (para cada 100 mil habitantes-ano); taxa de mortalidade infantil (mil nascidos vivos) e nascidos vivos de baixo peso (100 nascidos). As taxas de mortalidade foram calculadas com dados do SIM (óbitos) e do IBGE (população), em ambos os casos foram utilizadas a média dos anos de 2006 a 2010. Do SINASC, foram extraídos o número de nascidos vivos e o número de nascidos vivos de baixo peso para a média dos anos de 2006 a 2010.

A estatística descritiva foi realizada pela média, desvio-padrão das variáveis contínuas e frequência relativa da variável categórica porte populacional. Utilizou-se Anova para as associações bivariadas (Material Suplementar: http://cadernos.ensp.fiocruz.br/site/public_site/arquivo/csp-1501-17mate rialsuplementar_4497.pdf). Análises preliminares mostraram que muitas relações não eram lineares, assim, para facilitar a interpretação, todas as variáveis explicativas foram categorizadas. Categorizouse por quintis ou pontos de corte específicos. Porte populacional também seguiu as categorias do IBGE. Apenas as variáveis proporção de ESB por ESF e proporção de NASF por ESF ficaram com $\mathrm{n}=445$, pois, no estado, há 51 municípios sem nenhuma equipe de ESF.

Para análise ajustada, foi utilizada a regressão linear múltipla, sendo avaliados o ajuste do modelo e o diagnóstico de resíduos. Inicialmente, as variáveis foram modeladas dentro de blocos de variáveis semelhantes, e aquelas que apresentaram valor de $\mathrm{p}$ significativo $(\leq 0,05)$ foram incluídas no modelo final. No modelo final, foram retiradas variáveis não significativas a um nível de $\mathrm{p}>0,25$ usando a técnica stepwise backwards. A edição do banco e a análise dos dados foram realizadas utilizando o programa estatístico R versão 3.3.0 (http://www.r-project.org).

\section{Resultados}

Foram obtidos dados para todos os municípios, de forma que não há dados faltantes. A despesa municipal média em atenção primária à saúde realizada com recursos do Governo Federal no Rio Grande do Sul de 2011 a 2013 foi de $\mathrm{R} \$ 81,20$ por ano $(\mathrm{DP} \pm 35,50$; mínimo $=20,30$ e máximo $=213,30$ ). O estado possui 454 municípios com menos de 50 mil habitantes, ou seja, apenas 8,5\% dos municípios estão acima dessa faixa. Os municípios possuem, em média, 49,8\% (DP $\pm 1,3$ ) de mulheres, $16,1 \%$ 
$(\mathrm{DP} \pm 3,4)$ de idosos e 6,7\% (DP \pm 1,20) de crianças entre 0 e 5 anos. Em média, os municípios do Rio Grande do Sul possuem 4\% (DP \pm 4 ) de suas populações vivendo em extrema pobreza, sendo que esse percentual variou de 0 a 28,9\%. A renda domiciliar per capita média foi de R\$ 729,40 (DP $\pm 205,10)$, sendo o mínimo R\$ 325,50 e a renda máxima R\$ 1.722,40. Na análise bivariada, todas as variáveis apresentaram associação significativa com o desfecho, exceto o índice Gini (Tabela 1).

A análise ajustada pelas demais variáveis do bloco PAB fixo explica 39\% da variação dos gastos em atenção primária à saúde entre os municípios e constata-se que apenas as variáveis percentual da

Tabela 1

Descrição das variáveis contextuais dos municípios do Estado do Rio Grande do Sul, Brasil, por meio de medidas de tendência central e de dispersão ou frequências absoluta e relativa para variável porte populacional.

\begin{tabular}{|c|c|c|c|c|}
\hline Variáveis & Média ( \pm DP) & Mínimo & Mediana & Máximo \\
\hline Despesa com atenção primária à saúde per capita $(\mathrm{R} \$)$ & $81,20( \pm 35,50)$ & 20,30 & 83,90 & 213,30 \\
\hline PIB per capita $(\mathrm{R} \$)$ & $20.384,00( \pm 15.079,20)$ & $7.189,50$ & $16.807,40$ & $211.410,50$ \\
\hline Densidade demográfica (habitantes/km²) & $87,0( \pm 316,9)$ & 1,6 & 23,5 & $2.917,9$ \\
\hline Programa Bolsa Família (\% da população) & $10,7( \pm 11,1)$ & 0,0 & 7,5 & 45,8 \\
\hline Plano de saúde (\% da população) & $9,0( \pm 11)$ & 0,1 & 5,2 & 67,2 \\
\hline Extrema pobreza (\% da população) & $4,0( \pm 4)$ & 0,0 & 2,8 & 28,9 \\
\hline Taxa de ESF (100 mil habitantes-ano) & $26,4( \pm 17,4)$ & 0,0 & 27,9 & 135,0 \\
\hline Taxa de ACS (100 mil habitantes-ano) & $190,8( \pm 104,1)$ & 0,0 & 216,3 & 688,3 \\
\hline Proporção ESB por ESF & $0,7( \pm 0,4)$ & 0,0 & 1,0 & 1,0 \\
\hline Proporção NASF por ESF & $0,0( \pm 0,0)$ & 0,0 & 0,0 & 0,3 \\
\hline Renda domiciliar per capita ( $\mathrm{R} \$)$ & $729,40( \pm 205,10)$ & 325,50 & 701,60 & $1.722,40$ \\
\hline IDHM & $71,4( \pm 4,1)$ & 58,7 & 71,7 & 80,5 \\
\hline Gini & $46,6( \pm 6,8)$ & 28,0 & 47,0 & 72,0 \\
\hline Escolaridade (\% da população) & $25,3( \pm 7,8)$ & 9,2 & 24,4 & 57,6 \\
\hline Mulheres (\% da população) & $49,8( \pm 1,3)$ & 44,7 & 49,8 & 53,6 \\
\hline Idosos acima de 60 anos (\% população) & $16,1( \pm 3,4)$ & 7,3 & 15,9 & 29,4 \\
\hline Crianças 0-5 anos (\% da população) & $6,7( \pm 1,20)$ & 3,5 & 6,8 & 12,1 \\
\hline IDSUS & $628,2( \pm 75,5)$ & 384,0 & 635,5 & 823,0 \\
\hline UBS (100 mil habitantes-ano) & $46,5( \pm 33,8)$ & 0,0 & 37,9 & 317,2 \\
\hline Hospitais (100 mil habitantes-ano) & $5,7( \pm 8,8)$ & 0,0 & 0,9 & 67,5 \\
\hline $\begin{array}{l}\text { Taxa de mortalidade por causas externas ( } 100 \mathrm{mil} \\
\text { habitantes-ano) }\end{array}$ & $65,3( \pm 20,2)$ & 0,0 & 63,5 & 144,3 \\
\hline $\begin{array}{l}\text { Taxa de mortalidade por diabetes mellitus ( } 100 \mathrm{mil} \\
\text { habitantes-ano) }\end{array}$ & $28,4( \pm 14,1)$ & 0,0 & 26,8 & 91,1 \\
\hline Taxa de mortalidade infantil (mil nascidos vivos) & $12,1( \pm 7,6)$ & 0,0 & 11,7 & 40,0 \\
\hline \multirow[t]{2}{*}{ Nascidos vivos de baixo peso (100 nascidos) } & $8,4( \pm 2,5)$ & 0,8 & 8,4 & 21,3 \\
\hline & $\mathbf{n}$ & $\%$ & & \\
\hline \multicolumn{5}{|l|}{ Porte municipal [Rio Grande do Sul] (habitantes) } \\
\hline Até 5.000 & 227 & 45,8 & & \\
\hline $5.001-10.000$ & 104 & 21,0 & & \\
\hline $10.001-50.000$ & 123 & 24,8 & & \\
\hline $50.001-100.000$ & 24 & 4,8 & & \\
\hline Mais de 100.001 & 18 & 3,6 & & \\
\hline
\end{tabular}

ACS: agentes comunitários de saúde; DP: desvio padrão; ESB: equipes de saúde bucal; ESF: Estratégia Saúde da Família; IDHM: Índice de Desenvolvimento Humano Municipal; IDSUS: Índice de Desempenho do SUS; NASF: Núcleos de Saúde da Família ; PIB: Produto Interno Bruto; USB: unidade básica de saúde.

Nota: o "n" total foi 496 exceto para as variáveis proporção ESB por ESF e proporção NASF por ESF em que o "n" foi 445. 
população vivendo em extrema pobreza e porte populacional foram estatisticamente significativas (Tabela 2). No bloco PAB variável, a análise ajustada pelas demais variáveis explica $82 \%$ da variação dos gastos em atenção primária à saúde entre os municípios e conferiu às variáveis taxa ACS, taxa ESF e proporção ESB por ESF significância estatística (Tabela 2). Observa-se que, a análise ajustada pelas demais variáveis do bloco das variáveis sociodemográficas explica $26 \%$ da variação do referido gasto, e as variáveis: renda domiciliar per capita, IDHM, escolaridade, percentual de mulheres, percentual de idosos e percentual de crianças foram significativas (Tabela 3). Além disso, a análise ajustada pelas demais variáveis nos blocos estrutura e desempenho e perfil epidemiológico explicam, respectivamente, $46 \%$ e $15 \%$ da variação dos gastos em atenção primária à saúde entre os municípios, as variáveis IDSUS, UBS, hospital, do primeiro bloco, e taxa de mortalidade por causas externas, taxa de mortalidade infantil e percentual de nascidos vivos de baixo peso, do segundo bloco, foram estatisticamente significativas (Tabela 4).

O modelo final ajustado com as variáveis significativas de cada bloco explica $83 \%$ da variação dos gastos em atenção primária à saúde entre os municípios (Tabela 5). Nesse modelo, verifica-se que municípios com maiores taxas de ESF por 100 mil habitantes possuem um gasto médio de $\mathrm{R} \$ 51,30$ per capita (IC95\%: 43,30; 59,20) a mais do que municípios com menores taxas, e essa diferença é estatisticamente significativa. Do mesmo modo, municípios com maiores taxas de ACS por 100 mil habitantes possuem um gasto médio de R\$33,50 per capita (IC95\%: 26,10; 41,00) a mais do que municípios com menores taxas, e essa diferença é estatisticamente significativa. O gasto em atenção primária à saúde nos municípios de maior IDSUS, nesse modelo, é, em média, R\$ 9,10 per capita (IC95\%: 4,10; $14,10)$ a mais do que nos municípios de menor IDSUS. Essa diferença também foi estatisticamente significativa apesar de resultar em menor diferença em Reais. Também o percentual da população vivendo em extrema pobreza foi significativo estando associado a um gasto per capita de $\mathrm{R} \$ 6,90$ (IC95\%: 1,90; 11,80). Os resíduos foram normalmente distribuídos, homocedásticos e sem associação com os valores preditos.

\section{Discussão}

Os achados deste estudo sugerem que os gastos em atenção primária à saúde baseiam-se, em grande parte, na adesão municipal aos programas federais do $\mathrm{PAB}$ variável, e não nos indicadores do $\mathrm{PAB}$ fixo. Além disso, quanto maior o porte populacional do município menor parece ser o gasto per capita em atenção primária à saúde, e isso é explicado pelas diferenças de cobertura dos programas do PAB variável. Ademais, não houve relação desse gasto com os fatores associados com a demanda em saúde, como o perfil demográfico e epidemiológico.

Como os resultados mostram, o modelo, apenas com as variáveis que compõem o PAB variável, explica $82 \%$ da oscilação das despesas médias em atenção primária à saúde, enquanto o modelo final com as variáveis significativas de cada bloco apresentou $\mathrm{R}^{2}$ de $83 \%$. Com isso, provavelmente, a melhor oportunidade que os gestores têm para aumentar o financiamento em atenção primária à saúde é aderir aos programas estabelecidos em nível federal. O valor total que pode ser obtido pelos programas do $\mathrm{PAB}$ variável vem se sobressaindo em relação ao $\mathrm{PAB}$ fixo 11 , com certa redução na autonomia municipal no uso de tais recursos. Desde a publicação da NOB 96, diversos autores vêm questionando a capacidade de tal norma em promover o princípio da descentralização, uma vez que condiciona grande parte do financiamento à adesão aos programas, tais como ESF e ACS, definidos centralmente 12,13,14. Entre os pressupostos da descentralização, estão a redistribuição de poder e de recursos e o estabelecimento de novas relações entre os níveis de governo e controle social 15. Para tanto, é preciso respeitar as distintas realidades sociais e sanitárias de cada município, garantindo a autonomia financeira a eles. Havendo recurso, esse deveria ser agregado ao teto financeiro, PAB fixo, assim, cada município, à sua moda, deveria apresentar resultados concretos na melhoria dos níveis de saúde e condições de vida da sua população ${ }^{12}$. São reconhecidas, no entanto, as dificuldades de muitos municípios brasileiros em gerir seus recursos, sejam por questões técnicas ou até mesmo por não utilizarem os recursos para a resolução dos problemas mais prevalentes e graves da população.

Diferenças municipais nos gastos foram trazidas neste estudo e explicadas pelas diferenças do PAB variável. Os municípios de pequeno porte do Rio Grande do Sul investem mais per capita em 


\section{Tabela 2}

Coeficientes de despesa média em atenção primária à saúde em regressão linear múltipla (IC95\%: intervalo de 95\% de confiança) para os blocos Piso de Atenção Básica (PAB) fixo e PAB variável no modelo para os municípios do Estado do Rio Grande do Sul, Brasil, no período de 2011 a 2013.

\begin{tabular}{|c|c|c|c|c|}
\hline Bloco/Variáveis/Categorias & Estimativa per capita $(\mathrm{R} \$)$ & IC95\% & Valor de $p$ & $\mathbf{R}^{2}$ \\
\hline \multicolumn{5}{|l|}{ PAB fixo } \\
\hline \multicolumn{5}{|l|}{ PIB per capita $(\mathrm{R} \$)$} \\
\hline Até $25.000,00$ & Referência & & 0,97 & 0,39 \\
\hline Mais de $25.000,00$ & 0,1 & $-6,7 ; 6,9$ & & \\
\hline \multicolumn{5}{|c|}{ Densidade demográfica (habitantes $/ \mathrm{km}^{2}$ ) } \\
\hline $1,6 \mathrm{~F} 12,0$ & Referência & & 0,51 & \\
\hline $12,0+19,9$ & 1,2 & $-6,9 ; 9,3$ & & \\
\hline $19,9+27,9$ & 0,1 & $-8,0 ; 8,3$ & & \\
\hline $27,9+60,0$ & $-1,2$ & $-9,4 ; 7,1$ & & \\
\hline $60,0+2.917,9$ & $-7,5$ & $-17,4 ; 2,3$ & & \\
\hline \multicolumn{5}{|c|}{ Programa Bolsa Família (\% da população) } \\
\hline $0,00+0,03$ & Referência & & 0,34 & \\
\hline $0,03+4,07$ & $-0,2$ & $-11,2 ; 10,8$ & & \\
\hline $4,07+11,80$ & 6,3 & $-5,2 ; 17,7$ & & \\
\hline $11,80+20,87$ & 3,2 & $-9,3 ; 15,8$ & & \\
\hline $20,87+45,76$ & $-3,0$ & $-16,8 ; 10,7$ & & \\
\hline \multicolumn{5}{|l|}{ Plano de saúde (\% da população) } \\
\hline $0,10+1,93$ & Referência & & 0,93 & \\
\hline $1,93+3,73$ & 0,1 & $-8,3 ; 8,4$ & & \\
\hline $3,73+6,92$ & 0,7 & $-7,9 ; 9,3$ & & \\
\hline $6,92+13,04$ & 0,6 & $-8,5 ; 9,8$ & & \\
\hline $13,04 \mathrm{~F} 67,22$ & $-3,1$ & $-13,6 ; 7,4$ & & \\
\hline \multicolumn{5}{|c|}{ Extrema pobreza (\% da população) } \\
\hline $0,00+0,58$ & Referência & & $<0,01$ & \\
\hline $0,58+1,88$ & 6,5 & $-2,6 ; 15,6$ & & \\
\hline $1,88+3,79$ & 2,8 & $-6,8 ; 12,3$ & & \\
\hline $3,79+6,88$ & 6,8 & $-3,3 ; 16,9$ & & \\
\hline $6,88+28,94$ & 21,7 & 10,$9 ; 32,5$ & & \\
\hline \multicolumn{5}{|c|}{ Porte municipal [Rio Grande do Sul] (habitantes) } \\
\hline Até 5.000 & Referência & & $<0,01$ & \\
\hline $5.001-10.000$ & $-22,4$ & $-29,3 ;-15,4$ & & \\
\hline $10.001-50.000$ & $-34,3$ & $-42,8 ;-25,7$ & & \\
\hline $50.001-100.000$ & $-46,1$ & $-62,8 ;-29,3$ & & \\
\hline Mais de 100.001 & $-45,6$ & $-65,3 ;-25,9$ & & \\
\hline \multicolumn{5}{|l|}{ PAB variável } \\
\hline \multicolumn{5}{|c|}{ Taxa de ACS (100 mil habitantes-ano) } \\
\hline $0,00+88,63$ & Referência & & $<0,01$ & 0,82 \\
\hline $88,63+180,24$ & 12,2 & 6,$5 ; 18,0$ & & \\
\hline $180,24 \mathrm{~F} 234,18$ & 24,5 & 17,$6 ; 31,5$ & & \\
\hline $234,18+273,93$ & 29,7 & 22,$4 ; 37,0$ & & \\
\hline $273,93+688,32$ & 37,2 & 29,$7 ; 44,6$ & & \\
\hline \multicolumn{5}{|c|}{ Taxa de ESF (100 mil habitantes-ano) } \\
\hline $0,00+8,28$ & Referência & & $<0,01$ & \\
\hline $8,28+22,40$ & 10,9 & 4,$9 ; 16,9$ & & \\
\hline $22,40+32,03$ & 24,1 & 16,$8 ; 31,3$ & & \\
\hline $32,03+41,37$ & 35,9 & 28,$2 ; 43,5$ & & \\
\hline $41,37+135,03$ & 55,6 & 47,$8 ; 63,3$ & & \\
\hline \multicolumn{5}{|l|}{ Proporção ESB por ESF } \\
\hline 0,00 & Referência & & $<0,01$ & \\
\hline $0,01+0,99$ & 3,9 & 0,$0 ; 7,8$ & & \\
\hline 1,00 & 7,6 & 3,$8 ; 11,5$ & & \\
\hline \multicolumn{5}{|l|}{ Proporção NASF por ESF } \\
\hline 0,00 & Referência & & 0,22 & \\
\hline 0,01 ou mais & 3,4 & $-2,1 ; 8,9$ & & \\
\hline
\end{tabular}

ACS: agentes comunitários de saúde; ESB: equipes de saúde bucal; ESF: Estratégia Saúde da Família; NASF: Núcleos de Saúde da Família; PAB: Piso de Atenção Básica; PIB: Produto Interno Bruto.

Nota: o "n" total foi 496 exceto para as variáveis proporção ESB por ESF e proporção NASF por ESF em que o " $n$ " foi 445. 
Tabela 3

Coeficientes de despesa média em atenção primária à saúde em regressão linear múltipla (IC95\%: intervalo de 95\% de confiança) para o bloco de variáveis sociodemográficas no modelo para os municípios do Estado do Rio Grande do Sul, Brasil, no período de 2011 a 2013.

\begin{tabular}{|c|c|c|c|c|}
\hline Variáveis/Categorias & Estimativa per capita (R\$) & IC95\% & Valor de $\mathrm{p}$ & $\mathbf{R}^{2}$ \\
\hline \multicolumn{5}{|c|}{ Renda domiciliar per capita ( $\mathrm{R} \$)$} \\
\hline $325,47+551,71$ & Referência & & $<0,01$ & 0,26 \\
\hline $551,71+642,21$ & $-12,8$ & $-22,6 ;-2,9$ & & \\
\hline $642,21+765,77$ & $-22,8$ & $-34,4 ;-11,1$ & & \\
\hline $765,77+903,20$ & $-25,8$ & $-39,0 ;-12,6$ & & \\
\hline $903,2+1.722,37$ & $-31,1$ & $-46,0 ;-16,3$ & & \\
\hline \multicolumn{5}{|l|}{ IDHM (*100) } \\
\hline $58,7+67,6$ & Referência & & $<0,01$ & \\
\hline $67,6+70,3$ & 4,8 & $-5,9 ; 15,5$ & & \\
\hline $70,3+73,0$ & 19,6 & 5,$8 ; 33,3$ & & \\
\hline $73,0 \vdash 75,1$ & 30,1 & 13,$8 ; 46,4$ & & \\
\hline $75,1+80,9$ & 34,2 & 15,$4 ; 53,0$ & & \\
\hline \multicolumn{5}{|c|}{ Mulheres (\% da população) } \\
\hline 44,70 F 48,77 & Referência & & $<0,01$ & \\
\hline $48,77 \vdash 49,50$ & $-2,8$ & $-11,6 ; 6,1$ & & \\
\hline $49,50+50,17$ & $-9,4$ & $-18,7 ;-0,1$ & & \\
\hline $50,17+50,91$ & $-19,2$ & $-29,3 ;-9,1$ & & \\
\hline $50,91+53,61$ & $-19,1$ & $-30,8 ;-7,5$ & & \\
\hline \multicolumn{5}{|l|}{ Gini $(* 100)$} \\
\hline $28+42$ & Referência & & 0,09 & \\
\hline $42+45$ & 10,2 & 1,$5 ; 18,9$ & & \\
\hline $45 \mathrm{~F} 48$ & 11,1 & 2,$2 ; 19,9$ & & \\
\hline $48+52$ & 7,3 & $-1,7 ; 16,2$ & & \\
\hline $52+72$ & 9,1 & $-0,4 ; 18,5$ & & \\
\hline \multicolumn{5}{|c|}{ Escolaridade (\% da população) } \\
\hline $9,15 \vdash 18,84$ & Referência & & $<0,01$ & \\
\hline $18,84+22,20$ & 5,7 & $-4,2 ; 15,5$ & & \\
\hline $22,20+26,56$ & $-3,9$ & $-15,7 ; 7,9$ & & \\
\hline $26,56+31,75$ & $-10,6$ & $-24,0 ; 2,9$ & & \\
\hline $31,75+57,63$ & $-25,2$ & $-42,3 ;-8,1$ & & \\
\hline \multicolumn{5}{|l|}{ Idosos (\% da população) } \\
\hline $7,32 \vdash 13,25$ & Referência & & $<0,01$ & \\
\hline $13,25+15,19$ & 16,1 & 6,$5 ; 25,7$ & & \\
\hline $15,19+16,89$ & 9,9 & $-0,3 ; 20,1$ & & \\
\hline $16,89+18,86$ & 17,8 & 6,$7 ; 28,8$ & & \\
\hline $18,86+29,40$ & 18,1 & 5,$5 ; 30,6$ & & \\
\hline \multicolumn{5}{|c|}{ Crianças 0-5 anos (\% da população) } \\
\hline Até $6 \%$ & Referência & & 0,13 & \\
\hline $6-8 \%$ & $-8,8$ & $-17,8 ; 0,2$ & & \\
\hline Mais de $8 \%$ & $-12,1$ & $-25,8 ; 1,7$ & & \\
\hline
\end{tabular}

IDHM: Índice de Desenvolvimento Humano Municipal.

Nota: o " $n$ " total foi 496 para todas as variáveis. 


\section{Tabela 4}

Coeficientes de despesa média em atenção primária à saúde em regressão linear múltipla (IC95\%: intervalo de 95\% de confiança) para os blocos sobre estrutura e desempenho e perfil epidemiológico no modelo para os municípios do Estado do Rio Grande do Sul, Brasil, no período de 2011 a 2013.

\begin{tabular}{|c|c|c|c|c|}
\hline Bloco/Variáveis/Categorias & Estimativa per capita $(\mathrm{R} \$)$ & IC95\% & Valor de $p$ & $\mathbf{R}^{2}$ \\
\hline \multicolumn{5}{|l|}{ Estrutura e desempenho } \\
\hline \multicolumn{5}{|l|}{ IDSUS (*100) } \\
\hline $384 \mathrm{~F} 562$ & Referência & & $<0,01$ & 0,46 \\
\hline $562+616$ & 14,8 & 7,$3 ; 22,3$ & & \\
\hline $616+653$ & 22,7 & 15,$2 ; 30,3$ & & \\
\hline $653+691$ & 30,1 & 22,$2 ; 38,0$ & & \\
\hline $691 \mathrm{H} 823$ & 42,5 & 34,$5 ; 50,6$ & & \\
\hline \multicolumn{5}{|l|}{ UBS (100 mil habitantes-ano) } \\
\hline $0,00+23,55$ & Referência & & $<0,01$ & \\
\hline $23,55 \mathrm{~F} 32,63$ & 12,0 & 4,$4 ; 19,5$ & & \\
\hline $32,63+43,43$ & 15,7 & 8,$1 ; 23,4$ & & \\
\hline $43,43+63,57$ & 22,0 & 14,$2 ; 29,9$ & & \\
\hline $63,57+317,21$ & 28,0 & 19,$7 ; 36,2$ & & \\
\hline \multicolumn{5}{|c|}{ Hospitais (100 mil habitantes-ano) } \\
\hline 0,00 & Referência & & $<0,01$ & \\
\hline $0,01+5,00$ & $-17,5$ & $-25,1 ;-9,9$ & & \\
\hline $5,01+10,00$ & $-5,4$ & $-13,8 ; 2,9$ & & \\
\hline $10,01 \mathrm{~F} 20,00$ & 2,5 & $-4,6 ; 9,6$ & & \\
\hline 20,01 ou mais & 12,3 & 3,$4 ; 21,2$ & & \\
\hline \multicolumn{5}{|l|}{ Epidemiológicas } \\
\hline \multicolumn{5}{|c|}{ Taxa de mortalidade por causas externas (100 mil habitantes-ano) } \\
\hline $0,0+50,0$ & Referência & & $<0,01$ & 0,15 \\
\hline 50,0 F 59,3 & $-11,3$ & $-20,6 ;-1,9$ & & \\
\hline $59,3+68,6$ & $-15,6$ & $-25,0 ;-6,1$ & & \\
\hline $68,6+80,2$ & $-10,7$ & $-20,1 ;-1,3$ & & \\
\hline $80,2+144,7$ & 2,4 & $-6,9 ; 11,8$ & & \\
\hline \multicolumn{5}{|c|}{ Taxa de mortalidade por diabetes mellitus (100 mil habitantes-ano) } \\
\hline $0,0+16,8$ & Referência & & 0,11 & \\
\hline $16,8+23,8$ & $-6,7$ & $-16,1 ; 2,6$ & & \\
\hline $23,8+30,9$ & $-7,8$ & $-17,1 ; 1,4$ & & \\
\hline $30,9+39,1$ & $-6,2$ & $-15,4 ; 3,0$ & & \\
\hline $39,1 \mathrm{~F} 91,5$ & 2,5 & $-6,7 ; 11,7$ & & \\
\hline \multicolumn{5}{|c|}{ Taxa de mortalidade infantil (mil nascidos vivos) } \\
\hline $0,0+6,4$ & Referência & & $<0,01$ & \\
\hline $6,4+10,2$ & $-9,5$ & $-18,9 ;-0,2$ & & \\
\hline $10,2+13,1$ & $-16,0$ & $-25,6 ;-6,4$ & & \\
\hline $13,1+17,9$ & $-9,5$ & $-19,0 ;-0,1$ & & \\
\hline $17,9+44,0$ & $-0,1$ & $-9,5 ; 9,4$ & & \\
\hline \multicolumn{5}{|c|}{ Nascidos vivos de baixo peso (100 nascidos) } \\
\hline $0,8+6,6$ & Referência & & $<0,01$ & \\
\hline $6,6+7,9$ & $-15,0$ & $-24,1 ;-5,9$ & & \\
\hline $7,9+8,9$ & $-24,8$ & $-33,8 ;-15,8$ & & \\
\hline $8,9+10,1$ & $-20,0$ & $-29,5 ;-10,6$ & & \\
\hline $10,1+21,3$ & $-13,1$ & $-22,3 ;-3,9$ & & \\
\hline
\end{tabular}

IDSUS: Índice de Desempenho do SUS; USB: unidade básica de saúde.

Nota: o "n" total foi 496 para todas as variáveis. 


\section{Tabela 5}

Coeficientes de despesa média em atenção primária à saúde em regressão linear múltipla (IC95\%: intervalo de 95\% de confiança) no modelo final para os municípios do Estado do Rio Grande do Sul, Brasil, no período de 2011 a 2013.

\begin{tabular}{|c|c|c|c|c|}
\hline Variáveis/Categorias & Estimativa per capita $(\mathrm{R} \$)$ & IC95\% & Valor de $p$ & $\mathbf{R}^{2}$ \\
\hline \multicolumn{5}{|c|}{ Taxa de ESF (100 mil habitantes-ano) } \\
\hline $0,00+8,28$ & Referência & & $<0,01$ & 0,83 \\
\hline $8,28+22,4$ & 9,3 & 3,$3 ; 15,4$ & & \\
\hline $22,4 \mathrm{~F} 32,03$ & 21,1 & 13,$7 ; 28,6$ & & \\
\hline $32,03+41,37$ & 32,2 & 24,$4 ; 40,0$ & & \\
\hline $41,37+135,03$ & 51,3 & 43,$3 ; 59,2$ & & \\
\hline \multicolumn{5}{|c|}{ Taxa de ACS (100 mil habitantes-ano) } \\
\hline $0,00+88,63$ & Referência & & $<0,01$ & \\
\hline $88,63 \mathrm{~F} 180,24$ & 12,7 & 7,$0 ; 18,3$ & & \\
\hline $180,24 \mathrm{~F} 234,18$ & 23,0 & 16,$1 ; 29,9$ & & \\
\hline $234,18+273,93$ & 26,8 & 19,$5 ; 34,1$ & & \\
\hline $273,93+688,32$ & 33,5 & 26,$1 ; 41,0$ & & \\
\hline \multicolumn{5}{|l|}{ Proporção ESB por ESF } \\
\hline 0,00 & Referência & & $<0,01$ & \\
\hline $0,01+0,99$ & 3,5 & $-0,5 ; 7,4$ & & \\
\hline 1,00 & 5,8 & 2,$0 ; 9,6$ & & \\
\hline \multicolumn{5}{|l|}{ IDSUS $(* 100)$} \\
\hline $384 \mathrm{~F} 562$ & Referência & & $<0,01$ & \\
\hline $562+616$ & 0,3 & $-4,3 ; 4,8$ & & \\
\hline $616+653$ & 2,7 & $-1,9 ; 7,3$ & & \\
\hline $653+691$ & 3,7 & $-1,1 ; 8,6$ & & \\
\hline $691+823$ & 9,1 & 4,$1 ; 14,1$ & & \\
\hline \multicolumn{5}{|c|}{ Extrema pobreza (\% da população) } \\
\hline $0,00+0,58$ & Referência & & $<0,01$ & \\
\hline $0,58+1,88$ & $-1,4$ & $-5,7 ; 2,8$ & & \\
\hline $1,88+3,79$ & $-0,7$ & $-5,2 ; 3,7$ & & \\
\hline $3,79+6,88$ & 2,0 & $-2,8 ; 6,8$ & & \\
\hline $6,88+28,94$ & 6,9 & 1,$9 ; 11,8$ & & \\
\hline \multicolumn{5}{|c|}{ Escolaridade (\% da população) } \\
\hline $9,15+18,84$ & Referência & & 0,14 & \\
\hline $18,84+22,20$ & 4,5 & 0,$4 ; 8,6$ & & \\
\hline $22,20+26,56$ & 2,3 & $-2,0 ; 6,5$ & & \\
\hline $26,56+31,75$ & 0,4 & $-4,2 ; 4,9$ & & \\
\hline $31,75+57,63$ & 0,1 & $-5,1 ; 5,3$ & & \\
\hline
\end{tabular}

ACS: agentes comunitários de saúde; ESB: equipes de saúde bucal; ESF: Estratégia Saúde da Família; IDSUS: Índice de Desempenho do SUS.

Nota: o " $n$ " total foi 496 exceto para as variáveis proporção ESB por ESF em que o " $n$ " foi 445.

atenção primária à saúde do que os de maior porte, apesar de o financiamento por meio do PAB fixo considerar o número de habitantes do município. Autores apontam para a dificuldade de os municípios de grande porte ampliarem a cobertura de equipes de saúde da família, por conseguinte, não obtendo todos os recursos possíveis do PAB variável 16. Ademais, cidades pequenas, com menos de 20 mil habitantes, não possuem (ou possuem poucos) serviços de média e alta complexidade e terminam por orientar-se para a atenção primária à saúde 17 . Assim, como no presente estudo, a associação da despesa total com saúde e o porte populacional também foi fraca em estudo realizado com os municípios do Estado de Pernambuco 18. Entretanto, o aumento do porte populacional esteve relacionado 
com o aumento de gasto em saúde com recursos próprios em estudo que incluiu todos os municípios brasileiros 19 . Outro aspecto a ser considerado que diferencia os municípios é que aqueles com maior percentual de pessoas vivendo em extrema pobreza o gasto em atenção primária à saúde é maior. Diante do exposto, o percentual de pessoas vivendo em extrema pobreza revela-se como importante variável associada com o gasto em atenção primára à saúde, possivelmente associada com a demanda por serviços.

A presente pesquisa explorou a associação de diferentes indicadores com o gasto municipal em atenção primária à saúde. Os achados deste estudo sugerem que os aspectos para além dos indicadores sociodemográficos ou de necessidade em saúde podem ter influenciado na alocação de recursos na atenção primária à saúde entre os municípios do estado. Vários autores já trouxeram que fatores políticos, operacionais, administrativos, financeiros, estratégicos (oportunidades) e de interesse do controle social também podem influenciar a tomada de decisão 20,21,22,23. No entanto, é imprescindível o estabelecimento de parâmetros e indicadores, visto que abrange os princípios constitucionais do SUS 21. Estudos na Inglaterra também têm utilizado indicadores sociais, como o índice de Townsend, e de necessidade de saúde da população para avaliar a demanda por atenção primária à saúde e alocação de recursos 24,25. Nesse sentido, Ugá et al. 23 trazem, como ponto fundamental nas diferentes abordagens metodológicas para estimar uma distribuição equitativa dos recursos, incorporar, necessariamente, as desigualdades no perfil demográfico, segundo sexo e idade, e um indicador de necessidades, que permita dimensionar desigualdades relativas entre condições sanitárias e socioeconômicas das populações de distintas áreas geográficas, para tornar-se viável a implementação das ações resultantes das decisões tomadas 21 .

Como limitação, é pertinente ratificar que o estudo coletou dados sobre fonte de financiamento de base federal, e não foi contabilizado o fundo de tributação municipal e/ou repasse estadual. Essa decisão foi tomada baseada em estudos que mostram que o papel da esfera federal quanto ao financiamento ainda é preeminente em relação ao estado e municípios 26,27,28. Em adição, a possibilidade de erros de mensuração existe em algumas covariáveis, mas acredita-se que isso não seja associado ao desfecho, tendo um impacto menor nas estimativas, ou reduzindo a magnitude das associações pela introdução de um erro aleatório. Importante salientar que todas as informações foram obtidas de fontes oficiais/ governamentais, e o uso de tais bases de dados é importante também para auxiliar na identificação da validade e utilidade delas. Por outro lado, o presente estudo exploratório englobou 23 variáveis, sendo o maior estudo a relatar variáveis associadas a gastos específicos em atenção primária à saúde, dentro do conhecimento dos autores, visto que os estudos existentes trabalham com gasto per capita geral em saúde. Como a atenção primária à saúde é ordenadora do cuidado 29 , é essencial conhecer o processo de tomada de decisão em relação a ela para discutir a ampliação do direcionamento de recursos para esse bloco de serviços do setor saúde. Além disso, a inclusão de todos os municípios do estado elimina a possibilidade de viés de seleção.

Espera-se que os resultados apresentados instrumentalizem futuras discussões a cerca do rateio de recursos financeiros federais entre os municípios. Atualmente, os gastos estão atrelados à receita, cujos indicadores não parecem refletir totalmente a demanda que geram os gastos, isto é, o quadro sociodemográfico e epidemiológico. Além disso, outros indicadores que foram apresentados neste estudo possuem respaldo na literatura para serem utilizados de modo a tornar a distribuição do PAB fixo mais equitativo. Entretanto, a despeito do que é definido como PAB fixo em nível federal, outros estudos são necessários para estimar o valor per capita necessário para atender as demandas de saúde da população e conferir, de fato, maior autonomia financeira ao nível municipal de gestão. 


\section{Colaboradores}

F. S. Cabreira e R. K. Celeste participaram da concepção do estudo, da análise e interpretação de dados, revisaram criticamente o conteúdo, redigiram o estudo e aprovaram a versão final. F. Ritter colaborou na concepção do estudo, revisou criticamente o conteúdo e aprovou a versão final. V. R. Aguiar participou da interpretação de dados para o trabalho, redação, revisou criticamente o conteúdo e aprovou a versão final.

\section{Referências}

1. Starfield B. Is primary care essential? Lancet 1994; 344:1129-33.

2. Negri B. A política de saúde no Brasil nos anos 90: avanços e limites. Brasília: Ministério da Saúde; 2002.

3. Ministério da Saúde. Portaria no 1.602, de 9 de julho de 2011. Define o valor mínimo da parte fixa do Piso de Atenção Básica (PAB), para efeito do cálculo do montante de recursos a ser transferido do Fundo Nacional de Saúde aos Fundos de Saúde dos Municípios e do Distrito Federal. Diário Oficial da União 2011; 10 jul.

4. Andersen RM. National health surveys and the behavioral model of health services use. Med Care 2008; 46:647-53.

5. Almeida APSC, Nunes BP, Duro SMS, Facchini LA, Almeida APSC, Nunes BP, et al. Socioeconomic determinants of access to health services among older adults: a systematic review. Rev Saúde Pública 2017; 51:50.

6. Tomasi E, Nunes BP, Müller RM, Thumé E, Silveira DS, Siqueira FV, et al. Perfil de utilização de serviços de saúde por crianças de zona urbana no Brasil: estudo transversal de base nacional. Rev Bras Saúde Matern Infant 2015; 15:81-90.

7. Silva ZP, Ribeiro MCSA, Barata RB, Almeida MF. Perfil sociodemográfico e padrão de utilização dos serviços de saúde do Sistema Único de Saúde (SUS), 2003-2008. Ciênc Saúde Coletiva 2011; 16:3807-16.

8. Travassos C, Castro MSM. Determinantes e desigualdades sociais no acesso e na utilização de serviços de saúde. In: Giovanella L, Escorel S, Lobato LVC, Noronha JC, Carvalho AI, organizadores. Políticas e sistema de saúde no Brasil. Rio de Janeiro: Editora Fiocruz; 2008. p. 215-43.

9. Travassos C, Martins M. Uma revisão sobre os conceitos de acesso e utilização de serviços de saúde. Cad Saúde Pública 2004; 20 Suppl 2:S190-8.

10. Programa das Nações Unidas para o Desenvolvimento. Atlas do desenvolvimento humano no Brasil. Brasília: Programa das Nações Unidas para o Desenvolvimento; 2013.

11. Solla JJSP, Reis AAC, Soter APM, Fernandes AS, Palma JJL. Mudanças recentes no financiamento federal do Sistema Único de Saúde: atenção básica à saúde. Rev Bras Saúde Matern Infant 2007; 7:495-502.

12. Bueno WS, Merhy EE. Os equívocos da NOB/96: uma proposta em sintonia com os projetos neoliberalizantes? In: Conferência Nacional de Saúde On-Line/10a Conferência Nacional de Saúde. Brasília: Ministério da Saúde; 1997. p. 1-17.

13. Castro ALB, Machado CV. A política de atenção primária à saúde no Brasil: notas sobre a regulação e o financiamento federal. Cad Saúde Pública 2010; 26:693-705.

14. Vazquez DA. Efeitos da regulação federal sobre o financiamento da saúde. Cad Saúde Pública 2011;27:1201-12. 
15. Ministério da Saúde. Descentralização das ações e serviços de saúde: a ousadia de cumprir e fazer cumprir a lei. Brasília: Ministério da Saúde; 1993.

16. Portela GZ, Ribeiro JM. A sustentabilidade econômico-financeira da Estratégia Saúde da Família em municípios de grande porte. Ciênc Saúde Coletiva 2011; 16:1719-32.

17. Scatena JHG, Viana ALd'A, Tanaka OY. Sustentabilidade financeira e econômica do gasto público em saúde no nível municipal: reflexões a partir de dados de municípios mato-grossenses. Cad Saúde Pública 2009; 25:2433-45.

18. Espírito Santo ACG, Fernando VCN, Bezerra AFB. Despesa pública municipal com saúde em Pernambuco, Brasil, de 2000 a 2007. Ciênc Saúde Coletiva 2012; 17:861-71.

19. Araújo CEL, Gonçalves GQ, Machado JA, Araújo CEL, Gonçalves GQ, Machado JA. Os municípios brasileiros e os gastos próprios com saúde: algumas associações. Ciênc Saúde Coletiva 2017; 22:953-63.

20. Aguiar VR, Celeste RK. Necessidade e alocação de laboratórios regionais de prótese dentária no Brasil: um estudo exploratório. Ciênc Saúde Coletiva 2015; 20:3121-8.

21. Tanaka OY, Tamaki EM. O papel da avaliação para a tomada de decisão na gestão de serviços de saúde. Ciênc Saúde Coletiva 2012; 17:821-8.

22. Travassos C, Viacava F, Fernandes C, Almeida CM. Desigualdades geográficas e sociais na utilização de serviços de saúde no Brasil. Ciênc Saúde Coletiva 2000; 5:133-49.
23. Ugá MA, Piola SF, Porto SM, Vianna SM. Descentralização e alocação de recursos no âmbito do Sistema Único de Saúde (SUS). Ciênc Saúde Coletiva 2003; 8:417-37.

24. Gulliford MC, Jack RH, Adams G, Ukoumunne OC. Availability and structure of primary medical care services and population health and health care indicators in England. BMC Health Serv Res 2004; 4:12.

25. Baker D, Hann M. General practitioner services in primary care groups in England: is there inequity between service availability and population need? Health Place 2001; 7:67-74.

26. Porto SM, Santos IS, Ugá MAD. A utilização de serviços de saúde por sistema de financiamento. Ciênc Saúde Coletiva 2006; 11:895910.

27. Arretche M. Financiamento federal e gestão local de políticas sociais: o difícil equilíbrio entre regulação, responsabilidade e autonomia. Ciênc Saúde Coletiva 2003; 8:331-45.

28. Marques RM, Mendes A. Atenção básica e Programa de Saúde da Família (PSF): novos rumos para a política de saúde e seu financiamento? Ciênc Saúde Coletiva 2003; 8:403-15.

29. Starfield B. Primary care: concept, evaluation and policy. New York: Oxford University Press; 1992. 


\section{Abstract}

The study's objective was to explore the association between the components of fixed and variable Minimum Basic Care (Portuguese: PAB), sociodemographic factors, epidemiological profile, and municipal spending in primary health care in Rio Grande do Sul State, Brazil. An ecological study in 496 municipalities (counties) in the state was carried out. Mean variable municipal spending from 2011 to 2013 from the financial block of primary health care, representing the actual spending with federal budget transfers, was based on data from the Management Report of the Strategic Management Support Room, and multiple linear regression was used. To adjust the model, variables were grouped in five blocks according to the study's objective. Mean spending on primary health care was BRL 81.20 ( $S D \pm 35.50)$ per inhabitantyear. The block of variables comprising the fixed $P A B$ component explained $39 \%\left(R^{2}=0.39\right)$ of the variability in spending between municipalities, while for the variable $P A B$ block, $R^{2}$ was 0.82 , in the sociodemographic block, $R^{2}$ was 0.26 , in the structure-performance block $R^{2}$ was 0.46 , and in the epidemiological profile block the $R^{2}$ was 0.15 . In the final model, the variable associated with the highest estimated values for spending on primary health care was the rate of family health teams. Municipalities with 135 to 41 teams per 100,000 inhabitant-years spend BRL 51.00 more per capita than municipalities with zero to 0 to 8 teams. Spending on primary health care appears to be linked more to federal induction than to factors associated with health care demand, such as the demographic and epidemiological profile of the municipalities in the state of Rio Grande do Sul,

Healthcare Financing; Financial Resources; Health Planning; Health Management; Primary Health Care

\section{Resumen}

Este trabajo examina la asociación entre componentes del Paquete de Atención Básica (PAB) fijo y variable, los factores sociodemográficos y el perfil epidemiológico con los gastos municipales en atención primaria de salud en Río Grande do Sul, Brasil. Fue realizado un estudio ecológico con 496 municipios de Río Grande do Sul. La variable gasto medio municipal desde el año 2011 a 2013 del bloque financiero de la atención primaria de salud, que representó los gastos efectivos con la transferencia de recursos federales, se extrajo del Informe Gerencial de la Sala de Apoyo a la Gestión Estratégica. Se utilizó un modelo de regresión lineal múltiple. Con el fin de ajustar el modelo, las variables se agruparon en cinco bloques de acuerdo con el objetivo del estudio. El gasto medio con atención primaria de salud fue BRL 81,20 $(D P \pm 35,50)$ por habitante-año. El bloque de variables que componen el PAB fijo explicó el 39\% $\left(R^{2}=0,39\right)$ de la variabilidad de gastos entre municipios, mientras que, en el bloque del PAB variable, el $R^{2} f u e 0,82$, en el bloque sociodemográfico, el $R^{2}$ fue 0,26, en el bloque de estructura-desempeño, el $R^{2}$ fue $0,46, y$, en el bloque de perfil epidemiológico, el $R^{2}$ fue 0,15. En el modelo final, la variable que estuvo asociada a mayores valores estimados de gasto con atención primaria de salud fue la tasa de equipos de salud de la familia. Los municipios con el número de equipos entre 135 y 41 por 100 mil habitantes-año poseen un gasto de BRL 51,00 per cápita más que municipios con el número de equipos entre 0 y 8 . El gasto en atención primaria de salud parece estar más vinculado a las políticas federales de inducción que a factores asociados con la demanda en salud, como el perfil demográfico y epidemiológico de los municipios de Río Grande do Sul.

Financiación de la Atención de la Salud; Recursos Financieros en Salud; Planificación en Salud; Gestión en Salud; Atención Primaria de Salud
Recebido em 29/Ago/2017

Versão final reapresentada em 15/Mai/2018

Aprovado em 21/Jun/2018 